\title{
Robust Midsagittal Plane Extraction from Coarse, Pathological 3D Images
}

\author{
Yanxi Liu, Robert T. Collins, and William E. Rothfus* \\ The Robotics Institute, Carnegie Mellon University, Pittsburgh 15213, USA, \\ yanxi,rcollins@cs.cmu.edu \\ ${ }^{*}$ University of Pittsburgh Medical Center, Pittsburgh, PA
}

\begin{abstract}
This paper focuses on the evaluation of an ideal midsagittal plane (iMSP) extraction algorithm. The algorithm was developed for capturing the iMSP from 3D normal and pathological neural images. The main challenges are the drastic structural asymmetry that often exists in pathological brains, and the sparse, nonisotropic data sampling that is common in clinical practice. A simple edge-based, cross-correlation approach is presented that decomposes the iMSP extraction problem into discovery of symmetry axes from 2D slices, followed by robust estimation of 3D plane parameters. The algorithm's tolerance to brain asymmetries, input image offsets and image noise is quantitatively measured. It is found that the algorithm can extract the iMSP from input 3D images with (1) large asymmetrical lesions; (2) arbitrary initial yaw and roll angle errors; and (3) low signal-to-noise level. Also, no significant difference is found between the iMSP computed by the algorithm and the midsagittal plane estimated by two trained neuroradiologists.

Keywords: midsagittal plane, asymmetry, robust estimation
\end{abstract}

\section{Introduction}

Healthy human brains exhibit an approximate bilateral symmetry with respect to the interhemispheric (longitudinal) fissure plane bisecting the brain, known as the midsagittal plane (MSP). However, human brains are almost never perfectly symmetric $[4,5,6]$. Pathological brains, in particular, often depart drastically from reflectional symmetry. For effective pathological brain image alignment and comparison (e.g. $[4,10,11]$ ) it is most desirable to define a plane of reference that is invariant for symmetrical as well as asymmetrical brain images and to develop algorithms that capture this reference plane robustly.

We define an ideal midsagittal plane (iMSP) as a virtual geometric plane about which the given 3D brain image presents maximum bilateral symmetry. Computationally, this plane is determined by taking the majority votes from both axial and coronal 2D slices based on a sound geometric analysis (Section 2.1). Factors that challenge the robustness of an iMSP algorithm include: (1) the intrinsic factor: the brains being imaged can be either bilaterally symmetric or drastically asymmetric; (2) the extrinsic factor(s): anisotropism, under-sampling, 
initial transformation errors and artifacts/noise can be introduced during the imaging process.

The well known Talairach framework is an anatomical landmark-based approach to define a 3D brain coordinate system [18]. Patient image to Talairach framework registration is difficult to achieve automatically since it relies on identifying 3D anatomical features that may not be obvious in the image. Furthermore, when the interhemispheric sagittal plane no longer lies on a flat surface due to normal or pathological deformation (Figure 1(a)), the interhemispheric medial plane is ill defined. In contrast, the iMSP is based on global geometry of the head and can be found using low-level image processing techniques. Furthermore, it remains well-defined in pathological cases, forcing a virtual left-right separation consistent with the location where an ideal midsagittal plane would be if not for the presence of local brain deformation (Figure 1(b)).

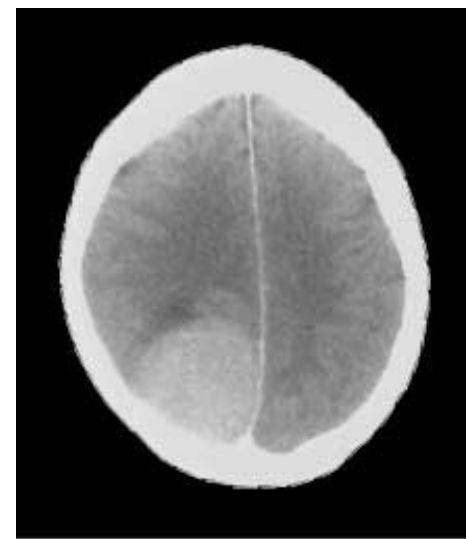

(a)

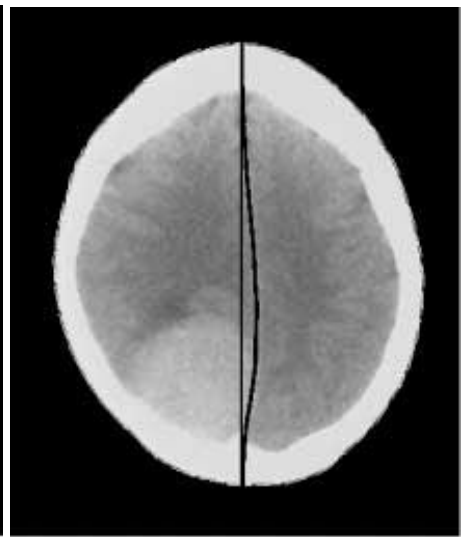

(b)

Fig. 1. (a) An axial brain slice, the midline is deformed due to a space occupying tumor. (b) The intersection of the extracted iMSP with the same 2D brain slice (straight line), and the deformed midline (curved line) captured by a "snake" active contour.

Designing a robust algorithm that deals with real clinical images originates from our desire to facilitate on-line clinical image database indexing and retrieval for real-time medical consultation $[10,11,12,13]$. We have developed a simple yet robust algorithm that can extract the iMSP of a brain from clinical CT or MR images (Section 2.2). The algorithm has been applied on more than $1003 \mathrm{D}$ clinical images and tested on both synthetic and real images with computed ground truth. Breakdown points of the iMSP extraction algorithm are found by varying brain orientation, lesion size, and noise level, and are compared against a maximization of mutual information method (Section 3). We also consider "ground truth" generated by human experts, and find no significant difference 
between the orientations estimated by our iMSP algorithm and those handpicked by two neuroradiologists (Section 3).

The goal of many existing MSP detection algorithms $[1,2,3,7,19]$ is to locate the plane of reflection of a roughly symmetrical brain image. Tolerance of these algorithms to asymmetry is reported to be low [3,7]. The MSP algorithm developed in [15] is tested on simulated PET images to show its insensitivity to focal asymmetries. More recently, Smith and Jenkinson [17] presented an algorithm for finding symmetry axes in partially damaged images of various modalities. However, no quantitative evaluations have yet been given and the computation is expensive.

\section{Ideal Midsagittal Plane Extraction}

We define an ideal head coordinate system centered in the brain with positive $X_{0}, Y_{0}$ and $Z_{0}$ axes pointing in the right, anterior and superior directions respectively (Figure 2, white coordinate axes). With respect to this coordinate system, the iMSP of the brain is defined as the plane $X_{0}=0$. Ideally, a set of axial (coronal) slices is cut perpendicular to the $Z_{0}\left(Y_{0}\right)$ axis, and the intersection of the MSP with each slice appears as a vertical line on the slice ${ }^{1}$.

In clinical practice, however, the imaging coordinate system XYZ (Figure 2 (a), black coordinate axes) differs from the ideal coordinates due to unintentional positioning errors and/or deliberate realignments introduced so that a desired volume can be better imaged, The orientation of the imaging coordinate system differs from the ideal coordinate system by three rotation angles, pitch, roll and yaw, about the $X_{0}, Y_{0}$ and $Z_{0}$ axes, respectively. The imaging coordinate system can also be offset (Figure 2(a)). The goal of an iMSP algorithm is to find the transformation between the two planes: $X_{0}=0$ and $X=0$.

\subsection{Geometry of the Ideal Midsagittal Plane}

Under the imaging coordinate system, the iMSP can be represented as

$$
a X+b Y+c Z+d=0
$$

where $(a, b, c)$ is a vector describing the plane normal. For the rest of this section we assume a nonzero scaling such that $\sqrt{a^{2}+b^{2}}=1$. Now consider the $i$ th axial slice, represented by the plane equation $Z=Z_{i}$. The symmetry axis of the $i$ th slice is the intersection of the above two planes: $a X+b Y+\left(c Z_{i}+d\right)=0$. This is the equation of a $2 \mathrm{D}$ line $\left(\theta_{i}, \rho_{i}\right)$ in the image $\mathrm{XY}$ plane, having line orientation $\theta_{i}=\arctan (b / a)$ and perpendicular offset from the image origin $\rho_{i}=c Z_{i}+d$. By examining this line equation, we can make two immediate observations. First, the orientation angle $\theta_{i}=\arctan (b / a)$ is the same for the symmetry axes of all slices regardless of their $Z_{i}$ position. Secondly, the offset

\footnotetext{
${ }^{1}$ The analysis given to the axial slices from now on can be applied to coronal slices (cut along the $\mathrm{Y}$ axis) as well with corresponding symbols changed: 'Z' to 'Y'.
} 


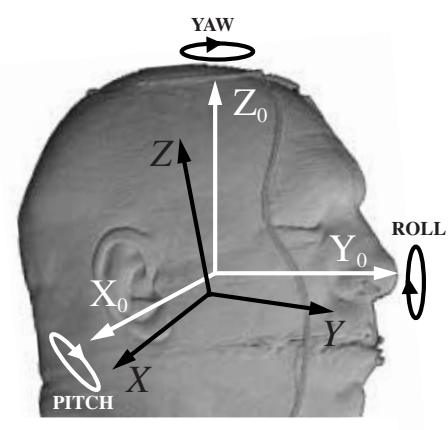

(a) $X_{0} Y_{0} Z_{0}$ v. $X Y Z$

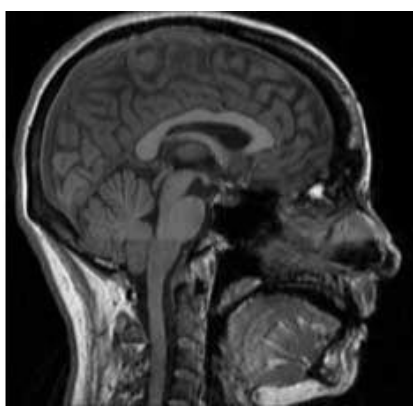

(b) $X_{0}=0$

Fig. 2. (a) Ideal head coordinate system $X_{0} Y_{0} Z_{0}$ vs. the imaging coordinate system $X Y Z$. The iMSP algorithm finds the transformation between planes $X_{0}=0$ and $X=0$. Rendered head courtesy of the Visible Human Project. (b) A midsagittal plane $\left(X_{0}=0\right)$ automatically extracted using our iMSP extraction algorithm.

$\rho_{i}$ of the symmetry axis on slice $Z=Z_{i}$ is linearly related to $Z_{i}$ as a function of plane parameters $c$ and $d$. Therefore, given the translational offset of at least two symmetry axes on different slices, we can estimate $c$ and $d$ by solving a set of linear equations. These observations form the basis of the iMSP extraction algorithm.

Under the ideal coordinate system, the iMSP passes through the origin and has normal vector $(1,0,0)$. Due to the scanning geometry, points in the ideal coordinate system are reoriented into the observed imaging coordinate by an unknown rotation $\mathcal{R}=\operatorname{yaw}(\theta) \operatorname{roll}(\phi) \operatorname{pitch}(\omega)$ and displaced by an unknown translation $\Delta X_{0}, \Delta Y_{0}$, and $\Delta Z_{0}$. Precisely, points in the ideal coordinate system are mapped into the imaging coordinate system by the transformation matrix

$$
\left[\begin{array}{l}
X \\
Y \\
Z
\end{array}\right]=\left[\begin{array}{cccc}
c \phi c \theta c \theta s \omega s \phi-c \omega s \theta & c \omega c \theta s \phi+s \omega s \theta & \Delta X_{0} \\
c \phi s \theta c \omega c \theta+s \omega s \phi s \theta & c \omega s \phi s \theta-c \theta s \omega & \Delta Y_{0} \\
-s \phi & c \phi s \omega & c \omega c \phi & \Delta Z_{0}
\end{array}\right]\left[\begin{array}{c}
X_{0} \\
Y_{0} \\
Z_{0}
\end{array}\right]
$$

where $c \theta \equiv \cos \theta, s \theta \equiv \sin \theta$, and so on. After some algebraic manipulation, the iMSP $X_{0}=0$ can be rewritten in terms of the imaging coordinate system as

$$
\cos \phi \cos \theta X+\cos \phi \sin \theta Y-\sin \phi Z-(n . \Delta)=0
$$

where $n=(\cos \phi \cos \theta, \cos \phi \sin \theta,-\sin \phi)^{T}$ is the unit normal vector of the plane and $\Delta=\left(\Delta X_{0}, \Delta Y_{0}, \Delta Z_{0}\right)^{T}$. Scaling appropriately, and comparing with (1), we find by inspection that

$$
a=\cos \theta \quad b=\sin \theta \quad c=-\tan \phi \quad d=-(n . \Delta) / \cos \phi .
$$

That is, the shared angle $\theta=\theta_{i}=\arctan (b / a)$ of each axial slice is actually the yaw angle of the head's imaging coordinate system. Furthermore, the roll angle 
$\phi$ can be determined from the offsets of the $2 \mathrm{D}$ symmetry axes on the set of slices by solving a linear system of equations $\rho_{i}=c Z_{i}+d$. Note from this equation and equation (3) that when the roll angle $\phi$ is zero, $c=0$, and thus all 2D symmetry axes have the same offset $\rho_{i}=d$; otherwise $\rho_{i}$ varies linearly from slice to slice. Finally, the quantity $(-d \cos \phi)$ measures the displacement of the imaging coordinate system in the direction normal to the symmetry plane.

In summary, if we can extract the 2D axes of reflection symmetry from a set of axial slices, we can completely determine the geometric equation $a X+b Y+$ $c Z+d=0$ of the ideal MSP. Furthermore, we can infer from this equation some of the 3D pose parameters of the patient's head with respect to the ideal head coordinate system, namely the yaw angle $\theta$, roll angle $\phi$ and the translational offset along the $X_{0}$ axis.

\subsection{Midsagittal Plane Extraction Algorithm}

The geometric results from the previous section have been used to develop an algorithm for automatically detecting the iMSP of a neural brain scan.

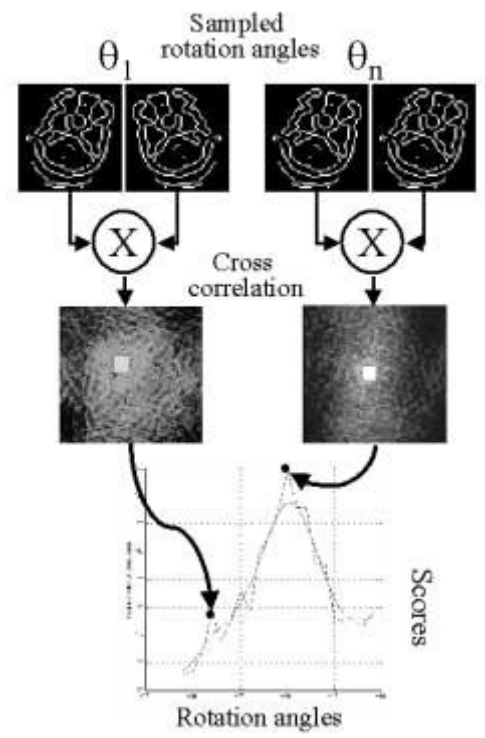

(a)

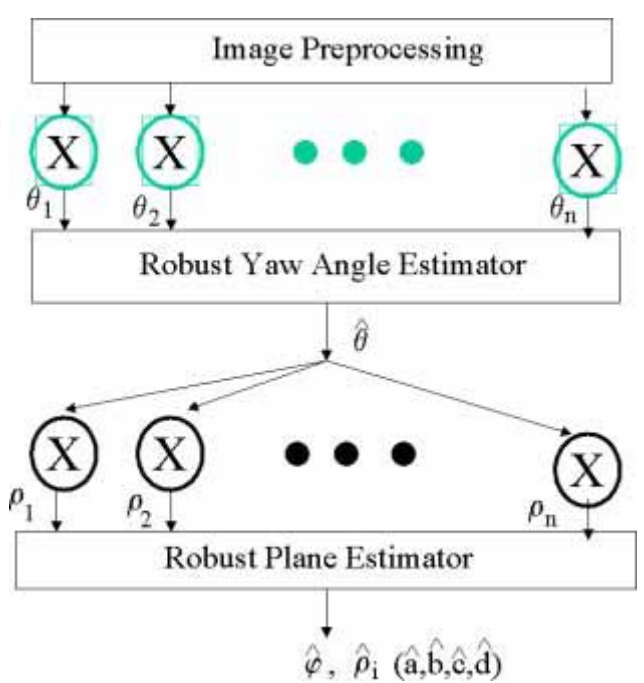

(b)

Fig. 3. (a) Using pairs of edge images (original image and its rotated reflection w.r.t. $X=0$ plane) to find the best correlation value for each given rotation angle. The brightest point indicates the highest correlation score. (b) A flow chart of the MSP extraction algorithm, where "X" with a circle around means $2 \mathrm{D}$ cross correlation, and with the addition of a square means multiple cross correlations of different rotated images. 
Input: a set of brain scans in axial (or coronal) format.

Output: head yaw and roll angles $\hat{\theta}, \hat{\phi}$, and translational offset of the iMSP from the $X_{0}=0$ plane.

\section{iMSP Extraction Algorithm ${ }^{2}$}

1. isolate the head region and compute binary edge images $S_{i}$.

2. find an estimated initial rotation error $\theta_{\text {init }}$ from one of the lowest $2 \mathrm{D}$ brain slices: for each $S_{i}$, construct $S_{i}^{\prime}=S_{i}$ reflected w.r.t. $X=0$ plane.

$\theta_{\text {init }}=\arg \max \left\{C_{i}\left(S_{i}, \operatorname{rot}\left(S_{i}^{\prime}, \theta\right)\right)\right\}$ where $C_{i}$ is the cross correlation of $S_{i}$ and rotated $S_{i}^{\prime}, \theta$ is sampled in the range of $\left[-90^{\circ}, 90^{\circ}\right]$ or $\left[-180^{\circ}, 180^{\circ}\right]$ if necessary.

3. find symmetry axis orientation $\theta_{i}$ on each $S_{i}$ (Figure $3(\mathrm{a})$ ):

for each $2 \mathrm{D}$ slice $S_{i}$,

for $\mathrm{j}=-10$ to 10 step 1 (degrees), $C_{i}\left(S_{i}, \operatorname{rot}\left(S_{i}^{\prime}, \theta_{\text {init }}+j\right)\right)$ end;

$\theta_{i}=\arg \max \left\{C_{i}\right\}$.

end;

4. compute the common yaw (or roll) angle (Figure 3(b)):

$\hat{\theta}=\operatorname{robust}\left(\theta_{1}, \cdots, \theta_{n}\right)$, where function robust eliminates outliers [20] and finds the median from weighted inliers.

5. compute image offsets $t_{i}$ by finding the maximum cross correlation value of each yaw (roll)-angle-corrected 2D slice and its vertical reflection.

6. robust estimation of image offsets $\rho_{i}$ : taking out outliers from $t_{i}$ using least median of squares [16] and fit a plane to the inliers using $\rho_{i}=c Z_{i}+d$.

7. compute all plane parameters (Equation (3)).

The algorithm is implemented on an SGI O2 R10000, using a mixture of MATLAB and $\mathrm{C}$ subroutines. Total time for all algorithmic steps is roughly 7 minutes. No special attention has yet been paid to speeding up the code, except for using the fast Fourier transform for cross correlation computation. The algorithm has been applied to over 100 3D image sets (Table 1) with varying modalities.

One strategy used to increase robustness to large orientation errors is to use both axial and coronal slices simultaneously to estimate the yaw and roll angles (usually one of these sets of slices is measured directly, and the other is created by resampling the image volume). This is done because the accuracy of symmetry axis offset detection degrades when axial (coronal) slices have a roll (yaw) angle beyond 20 degrees. However, there is no such angular limit to estimating yaw (roll) angles from axial (coronal) slices, as these are in-plane rotations. Figures 4 and 5 show examples of extracted symmetry axes when there are obvious asymmetries in the head.

\section{Evaluation}

No obvious mishaps have been observed when applying the iMSP extraction algorithm to over 100 image sets with varying modalities and scan geometries. In this section we report a series of experiments that are carried out to test the

\footnotetext{
${ }^{2}$ For further details of the algorithm see [9]
} 
Table 1. A Sample of Input 3D Image Data

\begin{tabular}{|c|c|c|c|c|l|}
\hline Set & Modality & Form & Matrix & Voxel $\left(\mathrm{mm}^{3}\right)$ & Pathology \\
\hline 5 & MR (T1) & coron & $256 \times 256 \times 123$ & $0.9375 \times 0.9375 \times 1.5$ & Normal \\
\hline 17 & CT & axial & $686 \times 550 \times 9$ & $0.5 \times 0.5 \times 10$ & $\begin{array}{l}\text { Right thalamic } \\
\text { acute bleed }\end{array}$ \\
\hline 58 & CT & axial & $678 \times 542 \times 17$ & $\begin{array}{c}0.5 \times 0.5 \times 5(1-9) \\
0.5 \times 0.5 \times 10(10-17)\end{array}$ & $\begin{array}{l}\text { Frontal astrocytoma } \\
\text { high grade glial tumor }\end{array}$ \\
\hline 109 & CT & axial & $512 \times 512 \times 21$ & $0.4395 \times 0.0 .4395 \times 5(1-10)$ & Left parietal infarct \\
$0.4395 \times 0.4395 \times 8(11-21)$ & \\
\hline 110 & CT & axial & $512 \times 512 \times 24$ & $0.4297 \times 0.4297 \times 5$ & Normal \\
\hline
\end{tabular}

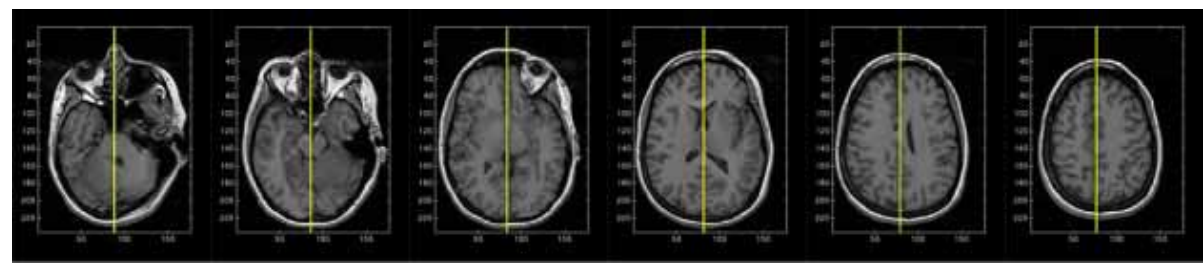

Fig. 4. The symmetry axes extracted from a set of axial slices with a 15 degree roll angle (out of plane rotation).

robustness of the iMSP algorithm. Two ground truth image test sets are created from datasets 5 and 110 respectively (Table 1), one is a sparse, axial CT volume and the other is a dense, coronal MR volume. Each ground truth test set is formed by finding the midsagittal plane by hand, then reflecting one half of the head volume about this midsagittal plane to overlay the other half, producing a perfectly symmetrical volume. Since the constructed test set is perfectly symmetric, the ground truth iMSP is known.

\section{Tolerance to initial offset errors}

To evaluate the accuracy of computed roll and yaw angles, the MR test image set was resampled using trilinear interpolation to artificially vary the yaw (roll) angles from -90 to 90 degrees in 5 degree intervals. The algorithm was then run on these sets to determine an estimated yaw and roll angle. In all cases, the error between estimated and actual angles is less than one degree. Given the image sampling interval, these errors are negligable.

\section{Tolerance to asymmetry}

The algorithm is tested by superimposing spherical "lesions" of varying density, size and position into the CT test image. The lesions are offset from the midline, resulting in pathological assymetry of the brain. The MSP algorithm's performance starts to decline when the tumor radius reaches 200 pixels $(85.94 \mathrm{~mm})$, 

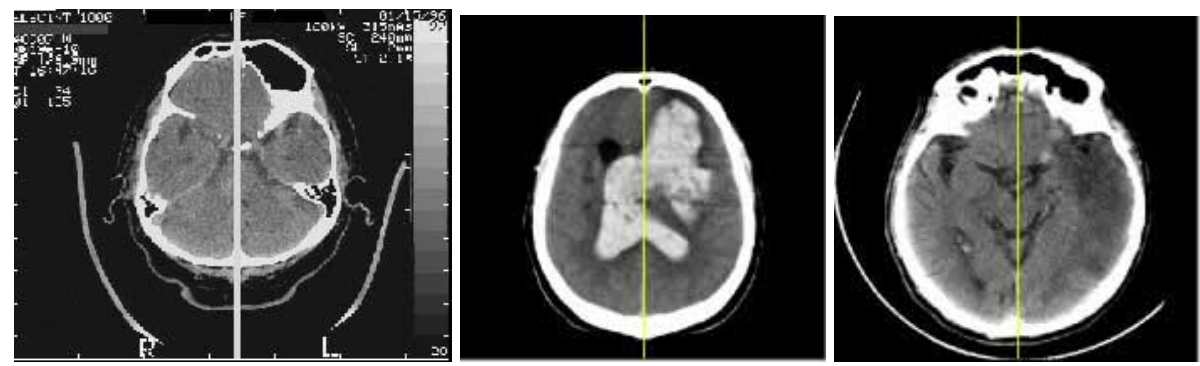

Fig. 5. The symmetry axes extracted from different CT scans where obvious asymmetry is present. Left to right: normal, acute blood, infarct.

and totally fails when the lesion radius reaches 250 pixels $(107.4 \mathrm{~mm})$ (Figures 6 and 7).

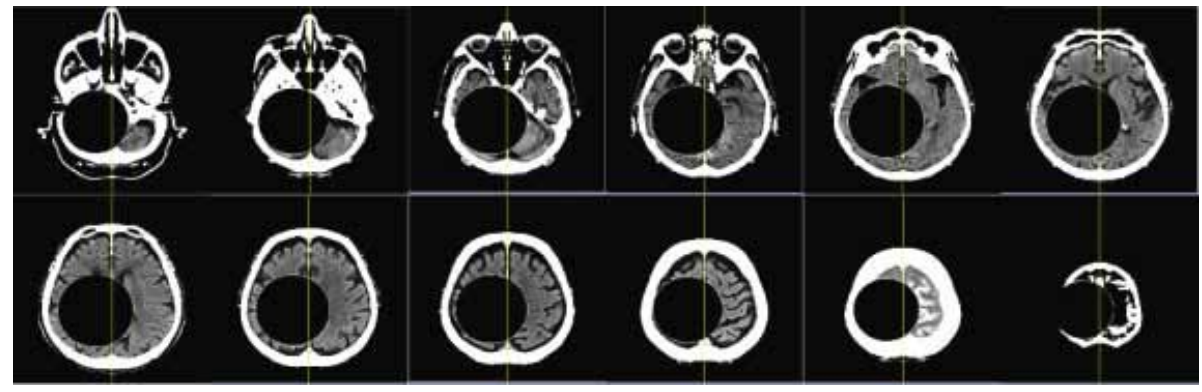

Fig. 6. The iMSP algorithm performs successfully when presented with an artificially grown lesion of 100 pixels $(42.97 \mathrm{~mm})$ radius.

\section{Tolerance to noise and asymmetry}

To study the effects of noise and asymmetry on the iMSP extraction algorithm, we have tested the algorithm on the MR ground truth dataset. The data is artificially degraded by adding different levels of zero-mean Gaussian noise, and by inserting spherical lesions of varying diameters. The algorithm breaking point is determined by incrementally adding noise until the algorithm fails to detect the correct symmetry plane. Each incremental addition of noise corresponds to a loss of $6.02 \mathrm{~dB}$ of SNR, or roughly 1 bit of information. ${ }^{3}$ Figure 8 shows representative slices, and iMSPs extracted by the algorithm. Naturally, the algorithm is more robust to noise when no lesion is present (Figure $8(\mathrm{~d})$ ). But the algorithm can handle very large levels of noise (up to $\mathrm{SNR}=-10.8 \mathrm{~dB}$ when no lesion is

\footnotetext{
${ }^{3}$ SNR or Signal to Noise Ratio is defined as $10 * \log (\operatorname{var}($ signal)/var(noise)). An SNR of less than 0 means that the noise has a higher variance than the signal.
} 

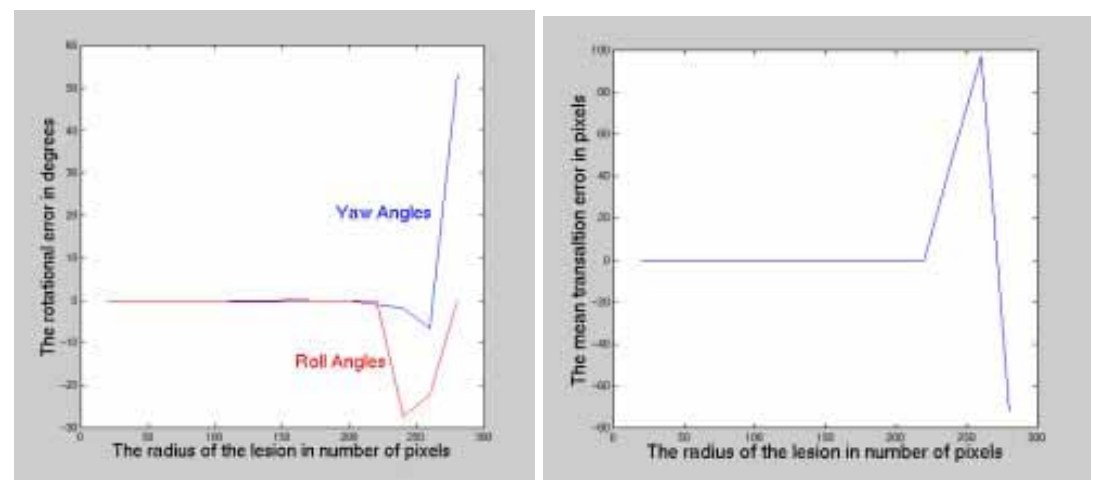

Fig. 7. Yaw and roll angle errors (Left) and translational offset errors (Right) versus the radius of an artificial lesion, in pixels ( 1 pixel $=0.4297 \mathrm{~mm}$ ). The spherical lesion is centered at $3 \mathrm{D}$ location $X=310 ; Y=210 ; Z=350$. The MSP algorithm's performance starts to decline when the tumor radius reaches 225 pixels $(96.68 \mathrm{~mm})$, and totally fails when the lesion radius reaches 250 pixels (107.43mm).

present), as well as large lesions (we tested with lesions of radius up to 60 pixels $=56.25 \mathrm{~mm})$.

\section{Comparison with a mutual information maximization method}

For comparison, an implementation of midsagittal plane extraction based on a general mutual information volume registration algorithm [14] was also tested. Volume registration can be used to identify an iMSP by finding the rigid transformation that best registers a volume with a version of itself reflected about the $X=0$ plane. The geometry underlying iMSP extraction in this case is analogous to that described in Section 2.2. We choose to compare a volume registration algorithm based on mutual information due to its intensity-based nature, in contrast to our edge-based method. The experimental results show that iMSP extraction based on mutual information registration breaks down at a lower level of noise than our algorithm, as shown in Figure 8. The volume registration algorithm had to be tested on the dense MR dataset, since it could not directly handle sparse, unisotropic clinical CT data.

Comparison with human experts To compare the algorithm with human performance, we had two neuroradiologists (one has 20 years experience, one is an intern) hand-draw the ideal midline on each $2 \mathrm{D}$ slice of several randomly chosen brain scans. The radiologists were allowed to view the whole set of the $2 \mathrm{D}$ slices from one volumetric image for reference while using a mouse to click on a computer screen directly. Although scan geometry tells us the angles of the symmetry axes on each axial slice should be the same (Section 2.1), there is a variation in the angles determined by the human expert. The standard deviation of the human measurement error on different sets of slices varies from 0.55 to 2.37 degrees (Table 2). 


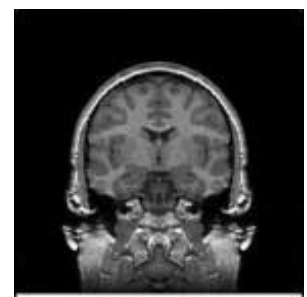

(a)

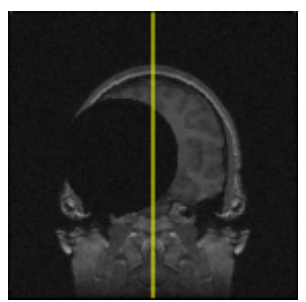

(b)

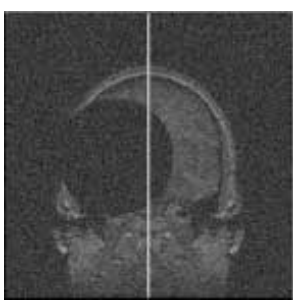

(c)

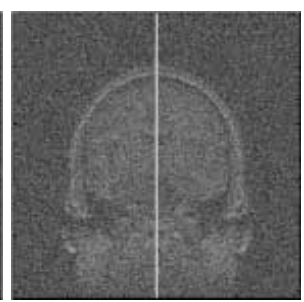

(d)

Fig. 8. Sample results on images artificially degraded with lesions and noise, to determine algorithm breaking points. (a) one slice from original dataset with no noise, $\mathrm{SNR}=43.35 \mathrm{~dB}$. (b) result from iMSP extraction based on mutual information registration, on a dataset with lesion plus noise. SNR of breaking point is $13.25 \mathrm{~dB}$. (c) result from our algorithm, on a dataset with lesion plus noise. SNR of breaking point is $-4.82 \mathrm{~dB}$. (d) result from our algorithm when run on a dataset with added noise only (no lesion). SNR of breaking point is $-10.84 \mathrm{~dB}$.

Table 2. Comparison of human vs. computer-estimated yaw angles (in degrees)

\begin{tabular}{|c|c|c|c|c|c|c|c|}
\hline Label & Pathology & \# Slices & $\begin{array}{l}\text { Expert 1 } \\
\text { Mean }\left(\theta_{i}\right)\end{array}$ & $\begin{array}{l}\text { Expert 2 } \\
\text { Mean }\left(\theta_{i}\right)\end{array}$ & $\begin{array}{l}\text { Expert 1 } \\
\operatorname{STD}\left(\theta_{i}\right)\end{array}$ & \begin{tabular}{|l} 
Expert 2 \\
$\operatorname{STD}\left(\theta_{i}\right)$
\end{tabular} & $\begin{array}{c}\text { Computed } \\
\text { Yaw } \hat{\theta}\end{array}$ \\
\hline \hline CMU121 & infarct & 17 & 1.6208 & 1.5730 & 1.0290 & 0.9972 & 0.8202 \\
\hline CMU126 & blood & 21 & 7.1404 & 6.2472 & 2.3678 & 0.5595 & 6.0347 \\
\hline CMU129 & infarct & 10 & -2.0248 & -1.8201 & 1.1141 & 1.6304 & -2.2318 \\
\hline CMU130 & blood & 21 & 1.3257 & 0.6829 & 0.9091 & 0.8967 & 1.3216 \\
\hline CMU170 & normal & 20 & -4.3187 & -4.5296 & 1.2781 & 2.0080 & -4.5109 \\
\hline CMU171 & normal & 22 & -1.3028 & -1.7582 & 1.0626 & 1.1963 & -1.0129 \\
\hline
\end{tabular}

\section{Discussion and Future Work}

In this paper, we have presented an iMSP extraction algorithm that is capable of finding the ideal MSP from coarsely sampled, asymmetrical neural images, without compromising accuracy on symmetrical ones. We have observed that iMSP computation using our algorithm is not adversely affected by lesions, mass effects, or noise in the images. This may seem strange since cross-correlation is used as a measure for the best matching of two images. It is natural to ask why the algorithm works so well on drastically asymmetrical images. We can provide the following relevant observations: 1. Majority rules: For a 3D pathological brain, a lesion only resides on a relatively small number of $2 \mathrm{D}$ slices, thus when the iMSP is fit to the whole set of $2 \mathrm{D}$ slices, normal slices with prominent bilateral symmetry dominate the iMSP's position. 2. Edge features: By using edge features rather than the original intensity images directly, the effect of strong density concentration around lesions is much reduced. 3. Lower brain slice stability: Lower brain slices are relatively stable due to the bilateral struc- 
ture of the skull. In practice, the lower brain slices are given more weight when determining the orientation of the iMSP. 4. Robust estimators: Robust estimation techniques are used to remove outliers from computed measurements before combining them to determine plane parameters.

We are currently exploring how to use the iMSP extraction algorithm to facilitate registration of pathological images with other modalities (PET, SPECT), and comparison of brain images (e.g. schizophrenia, acute infarct). Computing similarity among diverse brain images is part of an ongoing effort to study how symmetry-based features can be used for classification and retrieval of medically relevant cases $[10,11,12,13]$. We have also begun to apply the method on $3 \mathrm{D}$ pelvis images, to establish a common coordinate system for cross patient comparison, set an initial position for X-ray/CT registration [8], and evaluate left-right abnormality (Figure 9).
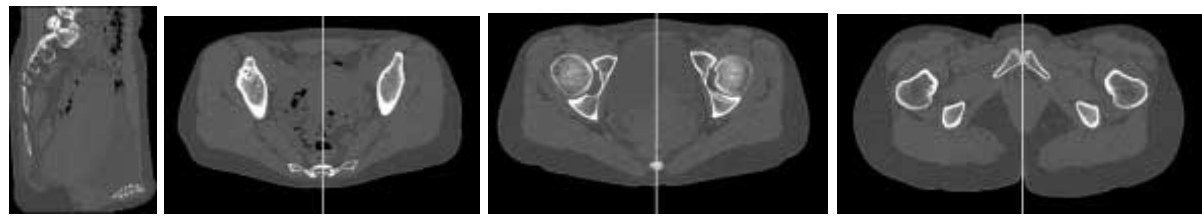

Fig. 9. Results from applying the MSP algorithm to pelvis CT images. Leftmost image is the midsagittal plane automatically extracted.

\section{References}

1. P. Allain, J.M. Travère, J.C. Baron, and D. Bloyet. Quantification of Brain Function. Tracer Kinetics and Image Analysis in Brain PET: Accurate PET positioning with reference to MRI and neuroanatomical data bases, pages 101,408. Elsevier Science Publishers, B.V., 1993.

2. B. Ardekani, J. Kershaw, M. Braun, and I. Kanno. Automatic detection of the mid-sagittal plane in 3-D brain images. IEEE Transactions on Medical Imaging, 16(6):947-952, December 1997.

3. M.E. Brummer. Hough transform detection of the longitudinal fissure in tomographic head images. IEEE Transactions on Medical Imaging, 10(1):74-81, March 1991.

4. T.J. Crow. Schizophrenia as an anomaly of cerebral asymmetry. In K. Maurer, editor, Imaging of the brain in psychiatry and related fields. Springer-Verlag, 1993.

5. R.J. Davidson and K. Hughdal, editors. Brain Asymmetry MIT Press/Bradford Books, Cambridge, Mass., 1996.

6. N. Geschwind and W. Levitsky, Human brain: left-right asymmetries in temporal speech region. Science, 161:186-187, 1968.

7. L. Junck, J.G. Moen, G.D. Hutchins, M.B. Brown, and D.E. Kuhl. Correlation methods for the centering, rotation, and alignment of functional brain images. Journal of Nuclear Medicine, 31(7):1220-1226, July 1990. 
8. D. LaRose et al. Post-operative measurement of acetabular cup position using x-ray/ct registration. In MICCAI2000, October 2000.

9. Y. Liu, R.T. Collins, and W.E. Rothfus. Robust Midsagittal Plane Extraction from Normal and Pathological 3D Neuroradiology Images. IEEE Transactions on Medical Imaging, in revision 2000.

10. Y. Liu and F. Dellaert. A Classification based Similarity Metric for 3D Image Retrieval. In Proceedings of Computer Vision and Pattern Recognition Conference, pages 800-805, June 1998.

11. Y. Liu and F. Dellaert. Classification Driven Medical Image Retrieval. In Image Understandard Workshop. DARPA, November 1998.

12. Y. Liu, F. Dellaert, and E.W. Rothfus. Classification driven semantic based medical image indexing and retrieval. Technical Report CMU-RI-TR-98-25, The Robotics Institute, Carnegie Mellon University, Pittsburgh, PA, 1998.

13. Y. Liu, W. Rothfus, and T. Kanade. Content-based 3d neuroradiologic image retrieval: Preliminary results. IEEE Workshop on Content-Based Access of Image and Video Libraries, in conjunction with International Conference of Computer Vision, pages 91,100, January 1998.

14. F. Maes, A. Collignon, D. Vandermeulun, G. Marchal, and P. Suetens. Multimodality image registration by maximization of mutual information. IEEE Transactions on Medical Imaging, 16(2):187,198, 1997.

15. S. Minoshima, K.L. Berger, K.S. Lee, and M.A. Mintum. An automated method for rotational correction and centering of three-dimensional functional brain images. Journal of Nuclear Medicine, 33(8):1579-1585, August 1992.

16. P.J. Rousseeuw. Least median-of-squares regression. Journal of the American Statistical Association, 79:871-880, 1984.

17. S. Smith and M. Jenkinson. Accurate robust symmetry estimation. In Medical Image Computing and Computer-Assisted Intervention - MICCAI'99, pages 308,317. Springer, 1999.

18. J. Talairach and P. Tournoux. Co-Planar Steriotaxic Atlas of the Human Brain. Thieme Medical Publishers, 1988.

19. J.P. Thirion, S. Prima, and G. Subsol. Statistical analysis of normal and abnormal dissymmetry in volumetric medical images. In Proc. of Workshop on Biomedical Image Analysis, pages 74-83. IEEE Computer Society, 1998.

20. Z. Zhang. Parameter estimation techniques: a tutorial with application on conic fitting. Image and Vision Computing, 15:59-76, 1997. 